\title{
Neutron spin filter based on dynamically polarized protons using photo-excited triplet states
}

\author{
T.R. Eichhorn ${ }^{\star a b}$, B. van den Brandt ${ }^{a}$, M. Haag ${ }^{a}$, P. Hautle ${ }^{a}$ and W.Th. Wenckebach ${ }^{a}$ \\ ${ }^{a}$ Paul Scherrer Institute, $\mathrm{CH}$ - 5232 Villigen PSI, Switzerland \\ ${ }^{b}$ Institute of Biological Systems, Ecole Polytechnique Fédérale de Lausanne, CH - 1015 \\ Lausanne, Switzerland \\ E-mail: tim.eichhorn@psi.ch
}

The use of polarized protons as a spin filter is an attractive alternative to the well-established neutron polarization techniques such as super mirrors and polarized helium-3 gas, since the large spin-dependent neutron scattering cross section for protons is useful up to the sub-MeV region. Nuclear spin order can be obtained by standard methods using dynamic nuclear polarization (DNP) through a coupling to highly polarized electron spins being at thermal equilibrium. This requires both low temperatures (ca. $1 \mathrm{~K}$ ) and strong magnetic fields $(2.5$ to $5 \mathrm{~T}$ ) in order to obtain a significant Boltzmann factor for the electron spin system. These rather strict conditions can be relieved by a more recent and very promising DNP method that uses short-lived electron triplet states in organic pentacene:naphthalene crystals. Photo-excitation of the triplet states provides a large electron spin order far beyond the thermal equilibrium. As a consequence the requirements for the cryogenic equipment and the magnetic field are relaxed significantly and technically simpler systems with open geometries are possible.

We have recently proven that the triplet DNP method can be used to build a reliably working neutron spin filter. It is operated in $0.3 \mathrm{~T}$ and about $100 \mathrm{~K}$ and has performed stably over periods of several weeks. So far we can report on high proton spin polarization values of up to 0.5 corresponding to an analyzing power of ca. 0.5 obtained with a sample of typical $5 \mathrm{~mm}$ thickness.

XVth International Workshop on Polarized Sources, Targets, and Polarimetry,

September 9-13, 2013

Charlottesville, Virginia, USA

${ }^{*}$ Speaker. 


\section{Introduction}

Polarized protons in solids are well-suited to be used as small-sized spin filter targets due to the strong spin interaction of neutrons and protons [1,2]. Proton polarization values up to almost unity have been achieved using dynamic nuclear polarization (DNP) [3]: by means of microwave irradiation close to the electron Larmor frequency in an external magnetic field, spin order of electrons is transferred dynamically to nuclei that experience a hyperfine coupling. Classical DNP schemes require significant experimental instrumentation to achieve low temperatures (ca. $1 \mathrm{~K}$ ) and strong magnetic fields $(2.5-5 \mathrm{~T})$ in which the electron spins are highly polarized in thermal equilibrium. Alternatively, an electron spin order far beyond thermal equilibrium can be established in a triplet state obtained by photo-excitation into an excited singlet state followed by intersystem crossing. Here the spin polarization results from selection rules of the latter process. In such an experiment [4] neither low temperatures nor high magnetic fields are required, both the apparatus is simplified and the accessibility to the filter target is improved, which is very attractive for applications.

\section{Polarized proton target as a neutron spin filter}

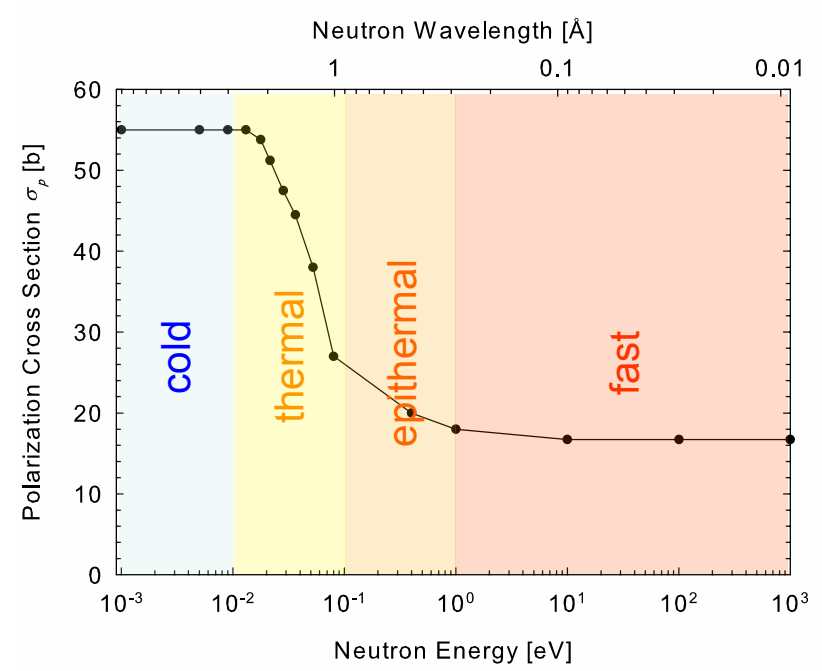

Figure 1: Typical dependency of polarization cross section $\sigma_{P}$ (given in barn) of solid hydrogenous materials as a function of the neutron kinetic energy. Experimental data is compiled from literature. Spin-dependent scattering is efficient up to the sub-MeV region.

The working principle of a polarized proton spin filter is based on spin-dependent scattering or absorption events that occur when a neutron beam passes a proton target. Since there is a large difference between the singlet and the triplet scattering cross section [5], the polarization of the proton target is imposed on the neutron beam: neutrons polarized anti-parallel to the protons will be much stronger scattered than those polarized parallel.

The striking feature of a proton target is its high filter efficiency in a broad energy range up to the sub-MeV region [6] that renders it superior to super mirror polarizers. Like a polarized helium-3 gas filter, a proton spin filter is practically independent of beam divergence. Additionally, 
the scattering cross section is nearly independent of the neutron wavelength. However, the actual implementation of a polarized proton spin filter has been restricted to a few special cases so far, e.g. for high precision neutron polarimetry [7, 8] or to polarize neutrons of thermal [9] and epithermal energy where it is the only method currently available [10]. This is probably due to the technical requirements of a classical DNP system, which can be significantly relaxed when employing the triplet DNP technique to realize a spin filter, what we recently demonstrated in a first test of principles [11].

In order to quantify the filter performance one can formally write down the effective cross section $\sigma$ of a proton target with polarization $P$, where one discriminates between the spin-independent part $\sigma_{0}$ and the spin-dependent part $\sigma_{P}$ (refer to fig. 1)

$$
\sigma_{ \pm}=\sigma_{0} \pm \sigma_{P} P
$$

and + and - are the two orientations of the neutron spin with respect to the target's polarization axis. Passing through the polarized proton target of proton density $N$ and thickness $d$ the two spin components of the neutron beam are attenuated by a factor $\exp \left(-\sigma_{ \pm} N d\right)$. Thus, an initially unpolarized neutron beam has a polarization behind the filter of

$$
A=\tanh \left(\sigma_{P} P N d\right)
$$

also referred to as the analyzing power. Due to the spin-independent scattering and a proton polarization lower than unity, the neutron intensity drops with a transmission factor

$$
T=\exp \left(-\sigma_{0} N d\right) \cosh \left(\sigma_{P} P N d\right)
$$

Note that for a given analyzing power the total transmission increases with the proton polarization.

\section{Dynamic nuclear polarization by photo-excited triplet states}

One among the systems most studied and appropriate for triplet DNP is crystalline naphthalene doped with a small concentration of pentacene. The ground state of pentacene is a nonparamagnetic singlet state. However, upon light irradiation pentacene can undergo intersystem crossing from an excited singlet state into an intermediate triplet state, which is paramagnetic. The triplet substates are non-degenerate at zero field and split further in an external magnetic field by the Zeeman interaction (refer to fig. 2). Using one canonical orientation $B \| X$ the two sites of pentacene doped in naphthalene are magnetically equivalent and only two ESR lines are observed (refer to fig. 3). In triplet DNP microwave irradiation occurs on one transition only and one can mathematically reduce the system to an fictitious spin- $\frac{1}{2}$ system.

The population rates to the triplet substates strongly favor the zero-field state $T_{X}$. For B ॥ X this state is conserved at any magnetic field values, and an effective spin polarization in the order of 0.9 can be reached, independent on the magnetic field strength and the temperature [12] . It is maintained during the lifetime of the triplet state in the order of $50 \mu \mathrm{s}$ which is short compared to the spin lattice relaxation time at cryogenic and room temperatures. However, the electron polarization has to be transferred quickly to the nuclei. 


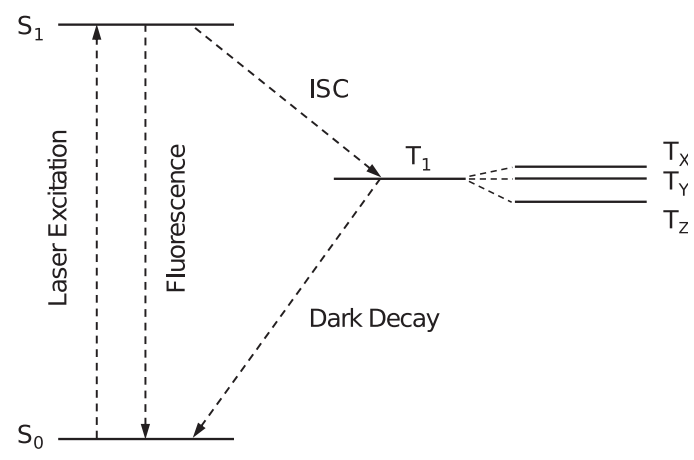

Figure 2: Jablonski diagram of the lowest electron energy levels of the pentacene molecule that are involved in the optical excitation process to provide the paramagnetic triplet state. The triplet substates, denoted as $T_{X}$, $T_{Y}$ and $T_{Z}$, are non-degenerate at zero field due to the dipolar coupling. As indicated, they are linked to the principal axes X,Y and Z of the pentacene molecule. Generally, in an external magnetic field, the zero field states are mixed, but if the magnetic field is oriented along one pentacene principal axis, the corresponding zero-field state is conserved. In praxis, the canonical orientation B $\|$ X conserves the pentacene state $T_{X}$ with the highest population, thus large spin order is maintained at any magnetic field value.

A polarization transfer scheme that has proven to be most efficient is the integrated solid effect (ISE) [13]. In this method simultaneous microwave irradiation and a magnetic field sweep over the whole ESR line in several ten's of $\mu$ s allows coherent polarization transfer within the triplet state lifetime $[14,15]$. As in classical DNP, the nuclear spin polarization is created locally around the paramagnetically activated pentacene molecule but can spread out further into the naphthalene host via spin diffusion. By repeating the cycle of triplet excitation and ISE transfer the proton spin polarization is accumulated and approaches the maximum value given by the triplet electron polarization. The proton spin lattice relaxation of crystalline naphthalene of high quality and pureness is around $24 \mathrm{~h}$ in a field of $0.3 \mathrm{~T}$ and a temperature of $100 \mathrm{~K}$. Depending on the pentacene doping ratio the proton polarization buildup time of a bulk sample can be between minutes and hours. As a consequence triplet DNP experiments need moderate cryogenic means only. High proton polarizations have been achieved in a field of only $0.3 \mathrm{~T}$ both at around $100 \mathrm{~K}$ and room temperature [16].

\section{Spin filter apparatus}

The apparatus is described in detail in [17]. Here, we briefly overview the key components: large pentacene:naphthalene crystals of high quality and uniformity are grown with a self-seeding vertical Bridgman technique [18]. Prior to the growth, naphthalene is extensively purified by zone refinement and pentacene is purified by re-sublimation growth [19]. The pentacene doping ratio is determined with optical transmission spectroscopy and evaluated to be typically between 2 and $4 \times 10^{-5}$. Using a polarizing microscope the crystallographic axes of the naphthalene host and the $\mathrm{X}$-axis of pentacene guest molecules are identified. Samples are cut into cubes of $5 \times 5 \times 5 \mathrm{~mm}^{3}$ with the three axes being the $\mathrm{X}-\mathrm{b}$ - and their normal axes.

The sample is mounted on a KelF holder with its bottom/top corresponding to the ac-plane and is introduced in a helium flow cryostat which is cooled to about $100 \mathrm{~K}$. The sample is irradiated 

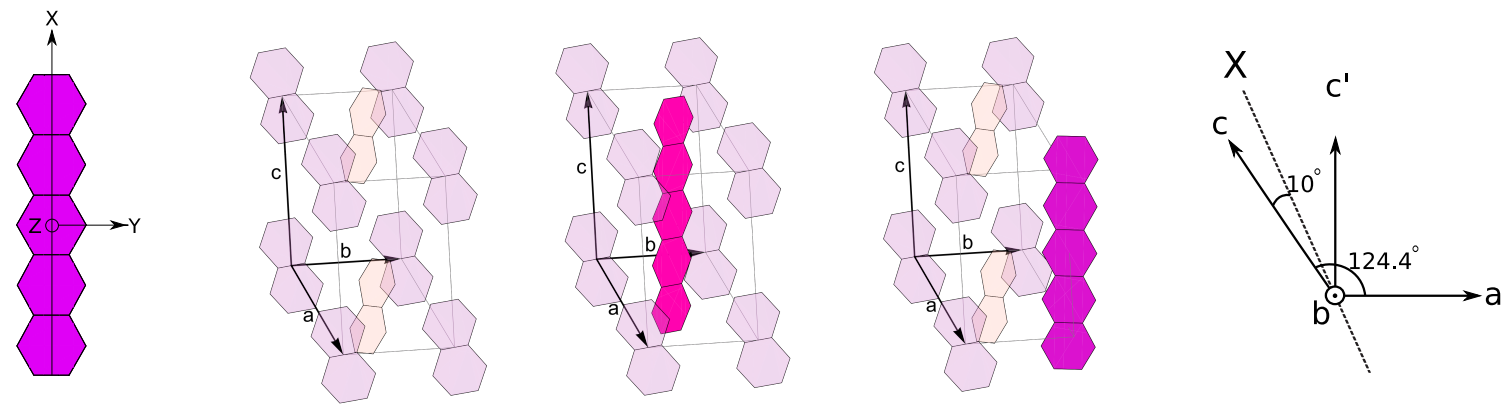

Figure 3: Crystallographic properties of pentacene:naphthalene crystals. From left to right: The pentacene molecule axes are the two in-plane axes $\mathrm{X}, \mathrm{Y}$ and the $\mathrm{Z}$ axis which is normal to the plane of the molecule. The unit cell of naphthalene is monoclinic with three axes $\mathrm{a}, \mathrm{b}$ and $\mathrm{c}$. The crystal symmetry is $\mathrm{P} 2_{1} / a$. Pentacene can be build into the naphthalene host at two different sites, replacing two naphthalene molecules. Both sites share the pentacene $\mathrm{X}$-axis. The length of the naphthalene axes are given with $\mathrm{a} \sim 8.1 \AA, \mathrm{b} \sim 5.9 \AA$ and $\mathrm{c}$ $\sim 8.6 \AA$. The a- and the c-axes are inclined by an angle of $\beta=124.4^{\circ}$. The pentacene $\mathrm{X}$-axis lies in the ac-plane with $10^{\circ}$ off the c-axis.

with green laser pulses at $515 \mathrm{~nm}$ that are generated as the second harmonics of a Yb:YAG disk laser (Jenlas IR50) and transported via a fiber to an optical stage at the bottom of the cryostat which collimates the unpolarized light to a beam of $6-7 \mathrm{~mm}$ waist shining along the b-axis of the sample crystal. In a typical experiment we use pulses of $400-500 \mathrm{~ns}$ length with a repetition rate of $400 \mathrm{~Hz}$ up to $4 \mathrm{kHz}$ and an energy of approximately $1 \mathrm{~mJ}$ per pulse at the entrance window of the cryostat. A static magnetic field of about $0.3 \mathrm{~T}$ is applied using a small electromagnet and DNP is performed with an X-band pulsed ESR system operating at $9.3 \mathrm{GHz}$ that generates the ISE passages synchronized to the laser pulses. The latter also enables us to observe the ESR signal of the triplet states and orient the static magnetic field along the $\mathrm{X}$-axis of the pentacene molecules. The proton polarization is measured both by NMR, where the enhanced signal is compared to the signal measured in thermal equilibrium, and by neutron transmission.

\section{Present status of spin filter performance}

The neutron spin filter apparatus has been operated and characterized at the BOA beam line of the continuous spallation neutron source SINQ at the Paul Scherrer Institute in Switzerland. In a first experiment we determined the wavelength-dependent polarization cross section $\sigma_{P}(\lambda)$ of crystalline naphthalene over the spectrum of the white cold beam [11]. As an alternative to NMR, this method provides the possibility to determine the proton polarization of our target sample with a high absolute precision of better than $3 \%$.

We have shown that the maximum proton polarization could be improved significantly by using fully deuterated pentacene instead of protonated pentacene [20]. It is possible to achieve a polarization of $P>0.40$ in about two hours. The highest value of $P=0.50$ was reached by reducing the DNP cycle repetition rate, thus lowering the heat input. This proton polarization corresponds to an analyzing power of $A \approx 0.5$ for a $5 \mathrm{~mm}$ thick filter and cold neutrons. We expect that the maximum filter polarization can be further increased by optimizing the DNP process and increasing the cooling efficiency of the target sample. 
A key point of the project is its implementation into a neutron optics system that provides the possibilities to focus a larger neutron beam onto the small aperture of the sample size using a confocal setup of parabolic mirrors. First promising tests show that it is possible to converge a 25 x $25 \mathrm{~mm}^{2}$ neutron beam to the size of the filter target of $5 \times 5 \mathrm{~mm}^{2}$. In the future different neutron optics concepts for standard scattering schemes will be evaluated by Monte Carlo simulations and the most favorable cases will be experimentally investigated.

\section{References}

[1] J.B. Hayter, G.T. Jenkin and J.W. White Phys. Rev. Lett. 33 (1974) 696.

[2] B. van den Brandt, H. Glättli, I. Grillo, P. Hautle, H. Jouve, J. Kohlbrecher, J.A. Konter, E. Leymarie, S. Mango, R.P. May, A. Michels, H.B. Stuhrmann and O. Zimmer Eur. Phys. J. B 49 (2006) 157.

[3] A. Abragam and M. Goldman Rep. Prog. Phys. 41 (1978) 395.

[4] A. Henstra, T.-S. Lin, J. Schmidt and W.Th. Wenckebach Chem. Phys. Lett. 165 (1990) 6.

[5] H. Glättli and M. Goldman Methods of Experimental Physics 23, part C (Academic Press, New York) (1987) 241.

[6] V.I. Lushchikov, Y.V. Taran and F.L. Shapiro Sov. J. Nucl. Phys. 10 (1970) 669.

[7] O. Zimmer, T.M. Müller, P. Hautle, W. Heil and H. Humblot Phys. Lett. B 455 (1999) 62.

[8] V.K. Aswal, B. van den Brandt, P. Hautle, J. Kohlbrecher, J.A. Konter, A. Michels, F.M. Piegsa, J. Stahn, S. van Petegem and O. Zimmer Nucl. Instrum. Methods Phys. Res. A 586 (2008) 86.

[9] S. Hiramatsu, S. Isagawa, S. Ishimoto, A. Masaike, K. Morimoto, S. Funahashi, Y. Hamaguchi, N. Minakawa and Y Yamaguchi J. Phys. Soc. Jpn. 45 (1978) 949.

[10] P.P.J. Delheij, J.D. Bowman, C.M. Frankle, D.G. Haase, T. Langston, R. Mortensen, S. Penttila, H. Postma, S.J. Seestrom and Y.F. Yen Nucl. Instrum. Methods Phys. Res. A 356 (1995) 120.

[11] M. Haag, T.R. Eichhorn, B. van den Brandt, P. Hautle and W.Th. Wenckebach Nucl. Instrum. Methods Phys. Res. A 87 (2012) 91.

[12] A.J. van Strien and J. Schmidt Chem. Phys. Lett. 70 (1980) 513.

[13] A. Henstra, T.S. Lin, J. Schmidt and W.Th. Wenckebach Chem. Phys. Lett. 165 (1990) 6.

[14] A. Henstra and W.Th. Wenckebach Mol. Phys. accepted for publication.

[15] T.R. Eichhorn, B. van den Brandt, P. Hautle, A. Henstra and W.Th. Wenckebach Mol. Phys. accepted for publication.

[16] M. Iinuma, Y. Takahashi, I. Shaké, M. Oda, A. Masaike and T. Yabuzaki Phys. Rev. Lett. 84 (2000) 171.

[17] T.R. Eichhorn, M. Haag, B. van den Brandt, P. Hautle, W.Th. Wenckebach, S. Jannin, J.J. van der Klink and A. Comment J. Magn. Reson. 234 (2013) 58.

[18] S. Selvakumar, K. Sivaji, A. Arulchakkaravarthi, N. Balamurugan, S. Sankar and P. Ramasam J. Crystal Growth 282 (2005) 370.

[19] R.A. Laudise, C. Kloc, P.G. Simpkins and T. Siegrist J. Cryst. Growth 187 (1998) 449.

[20] T.R. Eichhorn, M. Haag, B. van den Brandt, P. Hautle and W.Th. Wenckebach Chem. Phys. Lett. 555 (2013) 296. 